\title{
Effect of Acupuncture Stimulation of GV 20 Point on Electroencephalogram
}

\author{
Mehmet Tugrul Cabioglu1 ${ }^{* \#, ~ M u n i r e ~ K i l i n c ~ T o p r a k ~}{ }^{2 *}$, Derya Yılmaz ${ }^{3}$, Cagrı Cabıglu 4 \\ ${ }^{1}$ Department of Physiology, Medical Faculty, Başkent University, Ankara, Turkey \\ ${ }^{2}$ Department of Neurology, Medical Faculty, Başkent University, Ankara, Turkey \\ ${ }^{3}$ Department of Electrical and Electronics Engineering, Faculty of Engineering, Başkent University, \\ Ankara, Turkey \\ ${ }^{4}$ Department of Electrical and Electronics Engineering, Graduate School of Natural and Applied Sciences, \\ Middle East Technical University, Ankara, Turkey \\ Email: "tugcab@gmail.com
}

Received 13 May 2016; accepted 12 June 2016; published 15 June 2016

Copyright (C) 2016 by authors and OALib.

This work is licensed under the Creative Commons Attribution International License (CC BY). http://creativecommons.org/licenses/by/4.0/

(c) (i) Open Access

\begin{abstract}
Objective: This single-blind, randomized study aimed to evaluate effects of acupuncture stimulation at the GV 20 point on the electroencephalogram (EEG). Methods: Data was collected from 17 volunteers, aged 18 - 25 years old. EEG recordings were obtained before and after verum acupuncture and after a one-week interval EEG recording was also obtained before and after sham acupuncture. Spectral analysis of the EEG data was compared to baseline EEG. Results: Compared to baseline, there was an increase in the alpha band power at the T4 and T5 electrodes $(p<0.05)$ and a decrease in the beta band power at the T4, T6, and A2 electrodes following verum acupuncture. However, comparison of the data recorded before and after sham acupuncture revealed no significant differences. Conclusions: We claim that verum acupuncture stimulation at the GV 20 point has an effect on brain activity, which might be due to the known "mood modifying, relaxation effect" at that point.
\end{abstract}

\section{Keywords}

Acupuncture, Electroencephalography

Subject Areas: Internal Medicine

\section{Introduction}

For centuries, acupuncture has been used to treat numerous diseases and relieve pain [1]. In modern medicine,

\footnotetext{
${ }^{*}$ Contributed equally to this work.
}

${ }^{\#}$ Corresponding author.

How to cite this paper: Cabioglu, M.T., Toprak, M.K., Yılmaz, D. and Cabıoglu, C. (2016) Effect of Acupuncture Stimulation of GV 20 Point on Electroencephalogram. Open Access Library Journal, 3: e2653. 
acupuncture is considered to be an important tool in complementary medicine [2]. Although the scientific rationale and guidelines of acupuncture therapy have been summarized by the National Institutes of Health [3] [4], there are still some controversial issues about its efficacy, which can be seen in the publication of many systemic reviews revealing negative outcomes due to acupuncture [5].

Many studies have been performed to demonstrate the biochemical basis and mechanisms of action of acupuncture on the nervous system [1] [2] [6] [7]. According to these studies, mechanisms of action of acupuncture mostly involve endorphins and then the basic mechanism of its effect is theorized as "modification of the oscillations" in brain-body-mind function [8] [9].

Electroencephalogram (EEG) has previously been used to explore the effects of acupuncture [10]-[16]. However, as the design and methodology of the few published reports on humans vary largely from each other, they cause confusion rather than demonstrate a common mechanism of action for the effects of acupuncture on the electrical activity of the brain [17] [18]. Therefore, we designed this study to determine whether on-going EEG oscillations can be modulated by verum acupuncture stimulation of a traditional acupuncture point, GV 20, in comparison to placebo acupuncture of a sham point. We also aimed to determine if observed changes due to acupuncture were specific to certain EEG bands and if the supposed relaxation inducing effect of verum acupuncture of GV 20 point took place in human beings.

\section{Materials and Methods}

\subsection{Experimental Protocol}

In this single-blind, randomized study, we wanted to evaluate if any changes on human brain oscillations could be aroused by verum acupuncture of the Baihui (GV 20) point on EEG demonstrated by spectral analysis. We also wanted to compare these changes with the effects observed following the stimulation of a sham point. The selected acupuncture point, GV 20, is located at the midline of the head, $7 \mathrm{~cm}$ directly above the midpoint of the posterior hairline, at the midpoint of the line connecting the two auricles. The sham acupuncture point was determined as a point above Gb 8, which is not a known acupuncture point (Figure 1).

Seventeen subjects were randomly selected among volunteer male medical students aged 18 to 25 years, attending Baskent University, Faculty of Medicine University, Faculty of Medicine, following the approval of this project by the Local Ethics Committee. Candidates with a known neurological disease or those receiving regular medication were excluded. The subjects were naïve and had either none or little experience with acupuncture. The experiments were conducted with the consent of each subject.

It was imperative to follow a standard protocol for the preparation of subjects before and during EEG recording. Therefore, we arranged a relaxed and comfortable environment for the study subjects, ensuring that the subjects would not feel worried about the procedure and would remain alert during the study. We tried to overcome issues that could lead to changes in the EEG spectra that were irrelevant to acupuncture. Therefore, the subjects were informed about the procedure beforehand. Each volunteer attended two experimental sessions (acupuncture point stimulation and placebo point stimulation) separated by 1 week and all were blinded to the stimulation effect. They were asked to lie on a bed in a quiet room (mean temperature $20^{\circ} \mathrm{C}-22^{\circ} \mathrm{C}$ ) and rest for 15 minutes before experiments. All procedures were carried out with eyes closed and without any movement.

Acupuncture was performed by a professionally trained acupuncturist with 20 years experience in acupuncture. The acupuncturist has a national license. The stimulation was applied by inserting a thin needle at the GV

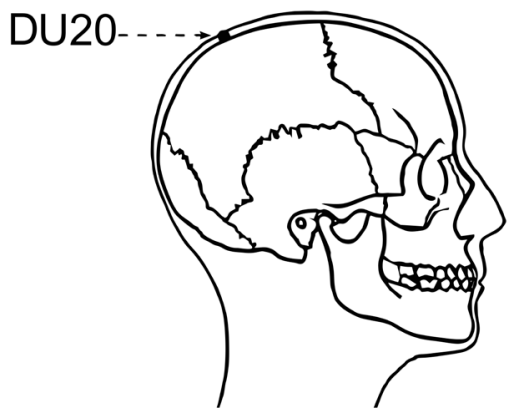

Figure 1. GV 20 point. 
20 acupoint $0.3-0.5$ inches subcutaneously. The needle remained at this point for 20 minutes and was then rotated with slight vertical back-and-forth movements resulting in a "de Qi" sensation (an ache, fullness, or heaviness with comfortable sensation such as alleviation). Placebo acupuncture was applied to a point below the Gb 8 point, which is not an acupuncture point (Figure 2). The same procedure that was applied at the verum acupuncture was used at the sham acupoint but without a resulting "de Qi" sensation.

A digital storage of 21-channel EEG was used. The EEG recordings were obtained using the Nicolet One system according to the international 10 - 20 system. Electrode impedance was kept close to $5 \mathrm{kOhm}$. The EEG signals were filtered using band pass filters set at $0.5-35 \mathrm{~Hz}$ and recorded at a sampling frequency of $500 \mathrm{~Hz}$. Acquired signals were amplified and inputted to the computer. During recordings, an input reference montage was used and the reference electrode was placed at the middle of the subject's forehead.

Spectral analysis to calculate the absolute power of EEG bands [Delta (0.5 - $4 \mathrm{~Hz}$ ), Theta (4 - $8 \mathrm{~Hz})$, Alpha (8 - $13 \mathrm{~Hz})$, Beta $(13-30 \mathrm{~Hz})$ ] for each electrode was carried out on the continuous segments of artefact-free EEG data, lasting for 1 minute duration, obtained before and after insertion of the acupuncture needle. After detrending the data, the EEG power spectrum was calculated with Fast Fourier Transform with 0.25 frequency resolution.

\subsection{Statistics}

Comparison of the baseline and verum/sham acupuncture stages and comparison of the baseline data of verum and sham acupuncture in terms of the calculated powers for each electrode were performed using the Statistical Package for the Social Sciences Software (SPSS Inc., Chicago, IL, USA) version 16.0. The Shapiro-Wilk test was used to test for normal distribution of the data. The paired Student's t-test and the non-parametric Wilcoxon $t$-test (Wilcoxon signed-rank test) were used to compare the normally and non-normally distributed data, respectively. The confidence level was set at $95 \%$. The power values of data with normal distribution are presented as mean \pm standard deviation and those with non-normal distribution are presented as median (range).

\section{Results}

The alpha and beta powers of the electrodes (in $\mu \mathrm{V}^{2}$ ) for verum and sham acupuncture stimulations are given in Table 1 and Table 2, respectively. The obtained statistical differences for verum acupuncture stimulation are bolded in Table 1 and given with numbered indices. Following verum acupuncture stimulation of the GV 20 point, in comparison to baseline, a statistically significant increase in the power of the alpha band at the T4 and T5 electrodes and a statistically significant decrease in the power of the beta band at the T4, T6 and A2 electrodes were observed $(p<0.05)$. Comparison of baseline data of verum and sham acupuncture groups revealed no significant difference.

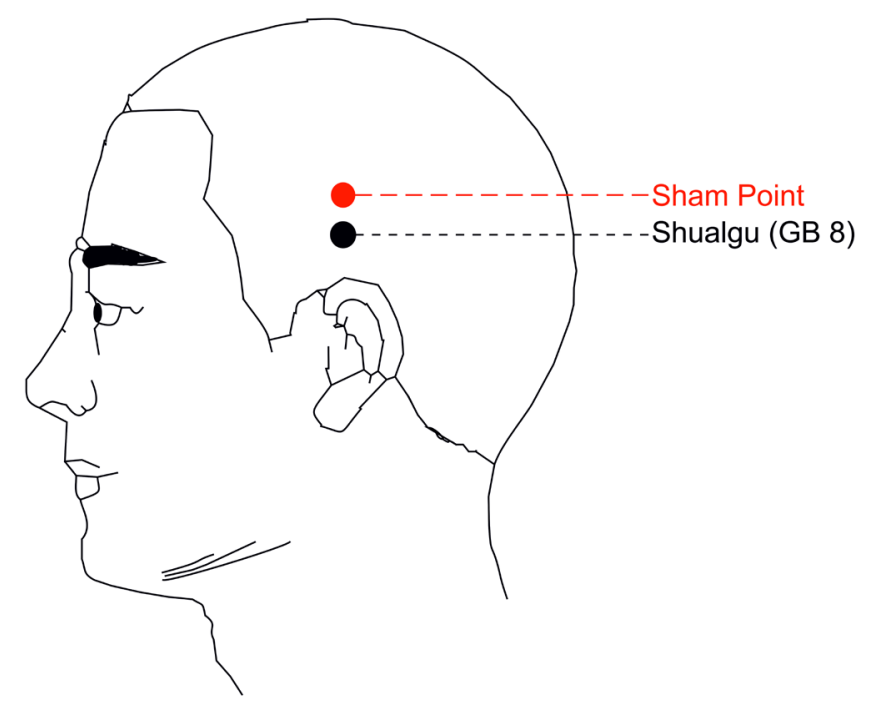

Figure 2. Sham point. 
Table 1. The alpha and beta powers of the electrodes $\left(\mu \mathrm{V}^{2}\right)$ and statistical differences for verum acupuncture stimulation.

\begin{tabular}{|c|c|c|c|c|c|c|}
\hline Band & Electrode & $\begin{array}{c}\text { Before insertion } \\
\text { of the needle (baseline) }\end{array}$ & $\begin{array}{l}\text { After insertion } \\
\text { of the needle }\end{array}$ & Band & $\begin{array}{c}\text { Before insertion } \\
\text { of the needle (baseline) }\end{array}$ & $\begin{array}{l}\text { After insertion } \\
\text { of the needle }\end{array}$ \\
\hline & Fp1 & $0.42(0.01-11.10)$ & $0.47(0.00-7.60)$ & & $0.95(0.00-11.51)$ & $0.56(0.00-7.92)$ \\
\hline & Fp2 & $0.56(0.12-10.38)$ & $0.57(0.13-7.64)$ & & $0.90(0.29-11.43)$ & $0.70(0.17-7.84)$ \\
\hline & F3 & $1.90(0.70-40.70)$ & $1.88(0.70-20.46)$ & & $2.37(1.12-18.36)$ & $1.89(1.14-11.33)$ \\
\hline & F4 & $2.14(0.59-23.07)$ & $2.21(0.69-14.15)$ & & $2.44(0.99$ - 9.04) & $2.04(1.12-8.28)$ \\
\hline & C3 & $5.29(1.57$ - 23.07) & $4.69(1.60$ - 29.53) & & $3.92 \pm 1.45$ & $3.86 \pm 1.77$ \\
\hline & C4 & $4.84(1.07-32.59)$ & $5.85(0.86$ - 35.88) & & $4.22(1.79-5.52)$ & $3.49(1.84-14.58)$ \\
\hline & P3 & $8.69(2.16-79.14)$ & $14.00(1.77-104.09)$ & & $4.50(1.95-8.65)$ & $3.48(2.16$ - 7.92) \\
\hline & $\mathbf{P 4}$ & $7.79(1.44-113.83)$ & $13.31(1.31$ - 137.68) & & $4.87 \pm 1.94$ & $4.46 \pm 1.99$ \\
\hline & 01 & $1.38(0.71-134.52)$ & $18.01(0.41-121.21)$ & & $6.41 \pm 3.19$ & $5.51 \pm 2.71$ \\
\hline & $\mathbf{O} 2$ & $1.08(0.54-200.95)$ & $21.61(0.08-276.07)$ & & $6.52(0.93-12.98)$ & $4.82(0.79-14.37)$ \\
\hline \multirow[t]{11}{*}{ Alpha } & F7 & $2.75(0.05-19.91)$ & $2.43(1.12-15.05)$ & Beta & $2.87(1.02-17.09)$ & $2.32(0.99-11.62)$ \\
\hline & F8 & $2.59(0.49-10.86)$ & $2.80(0.01-10.65)$ & & $2.48(0.52-12.43)$ & $1.95(0.94-7.02)$ \\
\hline & T3 & $4.69(1.90-25.41)$ & $5.14(2.81-26.76)$ & & $3.53 \pm 1.14$ & $3.17 \pm 1.03$ \\
\hline & T4 & $4.09(1.47$ - 28.96) & $5.17(1.49-33.71)^{\mathrm{a}}$ & & $3.36 \pm 1.03$ & $2.81 \pm 0.91^{\mathrm{c}}$ \\
\hline & T5 & $6.96(1.45-65.96)$ & $10.77(1.29-99.04)^{\mathrm{b}}$ & & $5.46(2.17-10.75)$ & $4.30(2.33-8.74)$ \\
\hline & T6 & $7.30(1.07$ - 129.20) & $12.00(0.87-178.42)$ & & $5.19(1.63$ - 9.97) & $3.67(1.64-9.53)^{d}$ \\
\hline & A1 & $0.85(0.00-22.16)$ & $0.87(0.00-17.39)$ & & $3.77(0.00-16.50)$ & $3.23(0.00-12.02)$ \\
\hline & A2 & 3.19 (0.39 - 15.58) & $2.80(0.67-26.50)$ & & $2.16(1.46-8.35)$ & $2.01(1.42-5.92)^{\mathrm{e}}$ \\
\hline & $\mathbf{F z}$ & $2.04(0.63-19.23)$ & $2.23(0.63-11.18)$ & & $1.93(0.96-12.22)$ & $1.51(1.2-8.86)$ \\
\hline & $\mathrm{Cz}$ & $4.60(1.24-25.22)$ & $5.27(1.25$ - 32.95) & & $4.06(2.16-6.50)$ & $3.44(1.97-11.83)$ \\
\hline & $\mathbf{P z}$ & $9.61(1.71-80.82)$ & $14.5(1.57-102.16)$ & & $4.90(1.84-8.41)$ & $4.54(2.22-10.89)$ \\
\hline
\end{tabular}

${ }^{\mathrm{a}}: \mathrm{z}=-2.012, p=0.044 ;{ }^{\mathrm{b}}: \mathrm{z}=-2.012, p=0.044 ;{ }^{\mathrm{c}}: \mathrm{t}=2.157, p=0.047 ;{ }^{\mathrm{d}}: \mathrm{z}=-2.201, p=0.028 ;{ }^{\mathrm{e}}: \mathrm{z}=-2.041, p=0.041$.

Table 2. The alpha and beta powers of the electrodes $\left(\mu \mathrm{V}^{2}\right)$ for sham acupuncture stimulation.

\begin{tabular}{|c|c|c|c|c|c|c|}
\hline Band & Electrode & Period 1 (baseline) & Period 2 & Band & Period 1 (baseline) & Period 2 \\
\hline \multirow{21}{*}{ Alpha } & Fp1 & $0.37(0.16-11.36)$ & $0.42(0.18-74.66)$ & \multirow{21}{*}{ Beta } & $0.70(0.26-10.60)$ & $0.79(0.36-15.89)$ \\
\hline & Fp2 & $0.43(0.05-12.72)$ & $0.43(0.15-79.82$ & & $0.69(0.09-12.47)$ & $0.75(0.33-18.92)$ \\
\hline & F3 & $1.47(0.76-12.03)$ & $1.84(0.92-127.29)$ & & $1.84(1.00-9.09)$ & $1.82(1.01-18.73)$ \\
\hline & F4 & $1.51(0.62-10.39)$ & $1.54(0.69-149.76)$ & & $1.83(0.79-8.39)$ & $2.19(0.73-17.69)$ \\
\hline & C3 & $3.33(0.21$ - 24.60) & $3.56(1.44-56.12)$ & & $3.46(1.62-9.03)$ & $3.65(1.67-10.44)$ \\
\hline & $\mathrm{C} 4$ & $3.30(1.25$ - 25.57) & $4.32(1.31$ - 31.55) & & $3.50(1.51-8.11)$ & $3.16(1.63-13.05)$ \\
\hline & P3 & $7.41(2.02-77.95)$ & $10.57(1.67-46.51)$ & & $5.04 \pm 2.67$ & $4.96 \pm 2.36$ \\
\hline & $\mathbf{P 4}$ & $5.04(2.04-91.53)$ & $7.88(2.01-47.60)$ & & $4.42(2.12-8.26)$ & $4.40(1.84-13.42)$ \\
\hline & 01 & $5.87(0.98-192.72)$ & $12.45(0.66$ - 97.90) & & $6.94 \pm 4.19$ & $6.14 \pm 3.19$ \\
\hline & $\mathbf{O} 2$ & $7.24(0.82$ - 253.34) & $17.97(0.79-188.71)$ & & $5.90(0.67$ - 16.94) & $4.73(0.77-17.53)$ \\
\hline & F7 & $1.84(0.85-7.60)$ & $1.85(0.83-66.22)$ & & $2.16(1.23-8.79)$ & $2.23(1.05-12.12)$ \\
\hline & F8 & $2.30(0.59-8.54)$ & $2.99(0.66$ - 53.43) & & $2.21(0.81-8.22)$ & $2.34(0.77-12.06)$ \\
\hline & T3 & $3.53(1.24-24.03)$ & 3.97 (1.38 - 28.49) & & $3.77(1.63-5.69)$ & $2.56(1.54-9.13)$ \\
\hline & T4 & $3.48(0.50-25.63)$ & $3.98(1.60$ - 16.54) & & $3.24 \pm 1.22$ & $3.22 \pm 1.51$ \\
\hline & T5 & $4.49(1.66$ - 100.09) & 9.10 (1.59 - 79.78) & & $5.52 \pm 2.85$ & $5.29 \pm 2.96$ \\
\hline & T6 & $5.17(0.86-127.40)$ & $8.49(1.22$ - 73.67) & & $4.74 \pm 2.04$ & $4.75 \pm 2.33$ \\
\hline & A1 & $1.79(0.71$ - 26.50) & $2.51(0.67-26.50)$ & & $3.90(2.35-44.70)$ & $3.98(1.75-44.81)$ \\
\hline & A2 & $1.4(0.45-19.62)$ & $1.97(0.44-17.91)$ & & $2.15(1.36-41.61)$ & $1.98(1.32-41.32)$ \\
\hline & $\mathbf{F z}$ & $1.53(0.60-10.70)$ & $1.49(0.66$ - 174.84) & & $1.37(0.87-8.40)$ & $1.70(0.83-16.32)$ \\
\hline & $\mathbf{C z}$ & $4.17(0.79-26.64)$ & $3.82(1.05-44.64)$ & & $3.29(1.44-9.18)$ & $3.32(1.54-14.61)$ \\
\hline & $\mathbf{P z}$ & $5.23(0.78-88.16)$ & $7.95(1.20-46.71)$ & & $4.58(2.26-8.04)$ & $4.98(2.19-15.11)$ \\
\hline
\end{tabular}




\section{Discussion}

In the present study, we investigated the effects of verum acupuncture at GV 20 and sham acupuncture on EEG findings of 17 healthy individuals before and after acupuncture. Our aim was to evaluate if any changes on human brain oscillations can arise by verum acupuncture at the GV 20 point demonstrated by spectral analysis of EEG. We wanted to compare these potential changes with the effects observed following placebo acupuncture at a sham point. We observed an increase in the power of the alpha band. GV 20 is a commonly used acupuncture point in neurological and psychiatric disorders [19]. The main therapeutic effects observed by acupuncture stimulation of GV 20 point are relief of headache, decrease in dizziness and anxiety, and relaxation. This point is chosen because verum acupuncture at points on meridians has been shown to activate certain areas of the brain [20] [21]. It is assumed that the acupuncture points, which are commonly used in clinical practice like the GV 20 point, might modulate a greater range of cortical areas than uncommonly used points. Furthermore, in clinical reports, acupuncture stimulation at the GV 20 point has been reported to generate a sedative effect.

EEG signals are multidimensional, non-stationary, time domain biological signals, externalizing information about processes going on in the brain, especially at the excitatory pyramidal neuron level. Since complex cortical generators continuously process a wide range of externally and internally generated stimuli and EEG signals are subject to noise and artefacts, extracting information from EEG is extremely challenging [22]. The two paradigms that have been used to analyze scalp EEG are segregation, including classification and clustering, and integration, including synchronization and coherence. According to these paradigms, in order to quantify EEG data, one can split the frequency spectrum into distinct bands, and with transformation of this data, calculate the power of these bands. EEG band power at a given electrode location reflects the micro-circuitry and functionality of the underlying pyramidal cells [23]. Traditional techniques, such as power spectrum analysis, have been used to analyse EEG data since 1900 and according to this technique, fixed bands can be used to divide the frequency spectrum of EEG data into delta, theta, alpha, and beta waves [i.e., the classical broad bands, delta (up to $4 \mathrm{~Hz}$ ), theta $(4-8 \mathrm{~Hz})$, alpha $(8-13 \mathrm{~Hz})$, beta $(13-25 \mathrm{~Hz})]$. In this study, we used power spectrum analysis to determine the changes in brain activity following verum/sham acupuncture stimulation in comparison to baseline.

After describing the methods we used in analyzing our EEG data, we will now review the literature pertinent to the effects of acupuncture on EEG. Changes in EEG observed by acupuncture manipulation in animals were previously reported in detail. According to a review on animal studies, needling of some common acupuncture points induced significant effects on the bioelectrical activity of the brain, while placebo acupuncture did not [13].

A number of studies with different protocols have been performed in humans to evaluate the electrophysiological effects of acupuncture [10]-[12] [14] [24]. However, as most of them used different protocols, they lead to confusion rather than clarifying the probable effects of acupuncture on human brain. For instance, while Rosted et al. [17] reported no changes in EEG after manual stimulation of the large intestine 4 (LI 4) acupuncture point, a more recent study demonstrated significant changes in EEG following verum acupuncture stimulation of the same point [14]. In another study, following transcutaneous electrical acupuncture stimulation of the LI 4 point, EEG changes were found to be correlated with relief of pain (decrease in pain score) [24]. According to this study, and some others, physiological responses to acupuncture are mediated by some special parts of the central nervous system, such as the forebrain, through the descending pathways to the autonomic nervous system [10] [11] [14] [15]. In some studies with negative results, subjects were kept alert using techniques, such as "asking subjects to silently countdown from 100 by sevens" or "asking subjects to fixate their eyes on a suspended clock” [17]. However, as these procedures demand concentration and calculation, they induce changes on EEG parameters. Therefore, such methods would be inappropriate to use in experimental settings that aim to track the changes in the mental states of study subjects. On the other hand, Rosted et al. [17] studied the effects of manual stimulation of the LI 4 point on EEG using only one electrode from Fpz to Oz. They concluded that acupuncture had no effect on the resting EEG in the frequency range of 2 - $30 \mathrm{~Hz}$. However, as the physiological or psychological effects of acupuncture often depend on stimulus parameters including site, area, intensity, and mode, studying only the changes taking place in only one point will be inadequate to draw conclusions. In another study, Chang et al. [10] investigated the effects of manual stimulation of the pericardium 6 acupuncture site on the EEG of the human brain. The EEG data were recorded before, during, and after sham/manual acupuncture. Spectral analysis of the data revealed a significant increase in the power of the alpha band during and 
after manual acupuncture. In addition, they reported that the frequency peaks in the alpha band of 12 channels were more synchronized during and after manual acupuncture. Streitberger et al. [14], who also studied the effects of verum acupuncture at the LI 4/sham point on the EEG findings of 20 healthy individuals, reported an increase in the frequency of the alpha band and a shift of the ratio of alpha/theta to the benefit of alpha band over all electrodes, when compared to sham acupuncture. Hence, while most studies analysing the effects of acupuncture on brain electrical activity lacked multiple EEG recordings or lacked control groups (comparisons of verum acupuncture to sham acupuncture) or were performed with inappropriate statistical analysis, which led to deceptive results, we tried to overcome these shortcomings by performing multiple EEG recordings with adequate electrode numbers and using a sham acupuncture group.

Randomized clinical trials have shown that activation of "pain control system" takes place following acupuncture applications resulting in various physiological changes in the autonomic nervous system including the enhancement of parasympathetic activity and suppression of sympathetic activity [15] [25]. Previous studies have also reported that acupuncture stimulation induces an increased level of plasma and CNS endogenous opioids like beta-endorphin, along with serotonin. It has been shown that increased levels of beta-endorphin, enkephalin, and serotonin result in a relaxing effect [14] [15]. It has also been accepted that acupuncture stimulation may induce the gastrointestinal, urinary, and genital system via activation of the autonomic nervous system. Although many acupoints have been used in the treatment of minor depression or anxiety up to now, the GV 20 point has always been the most common. We also assumed that enhancement of parasympathetic activity along with increased levels of CNS endogenous opioids and serotonin might have induced the documented increase in the power of alpha following GV 20 acupuncture point stimulation in our study (Table 3).

Ultimately, we concluded that acupuncture affected the electrical activity of the brain. The EEG findings obtained before and following acupuncture stimulation of the GV 20 point in this study, along with the results of spectral analysis, was due to a known effect of point, namely the "mood modifying", "soothing, relaxation" effect. We believe that detailed mechanisms for the therapeutic effects of acupuncture will come to light with advances in science. However, the imperative point should be the correct usage of scientific methods, along with the correct analysis of data.

There are important limitations in our study. Continuous heart rate and blood pressure were not recorded with EEG recordings. Collecting these points would have helped verify enhancement of the parasympathetic activity accompanying the increase in the alpha wave power and synchronization. These recordings would have offered greater support to the observed calming effect of verum acupuncture.

Table 3. Studies of EEG changes occurring due to acupuncture applications.

\begin{tabular}{|c|c|c|c|c|c|c|c|c|}
\hline & $\begin{array}{l}\text { Number of } \\
\text { Volunteers }\end{array}$ & Blindness & $\begin{array}{l}\text { Number of } \\
\text { Channels }\end{array}$ & $\begin{array}{l}\text { Stimulated } \\
\text { acupoints }\end{array}$ & $\begin{array}{l}\text { Stimulated } \\
\text { Sham Points }\end{array}$ & $\begin{array}{l}\text { Stimulation } \\
\text { Period }\end{array}$ & $\begin{array}{l}\text { Technique Used } \\
\text { For Analysis }\end{array}$ & Results \\
\hline $\begin{array}{c}\text { Rosted } \\
2001[17]\end{array}$ & 14 & Blinded & 1 & LI4 & Yes & Manual & $\begin{array}{l}\text { Spectral analysis } \\
\text { using FFT }\end{array}$ & $\begin{array}{l}\text { No significant } \\
\text { changes }\end{array}$ \\
\hline $\begin{array}{c}\text { Chen } \\
2006[11]\end{array}$ & 12 & Blinded & 124 & LI4 & Yes & EA & $\begin{array}{l}\text { Spectral analysis } \\
\text { using FFT }\end{array}$ & $\begin{array}{c}\text { Significant } \\
\text { change with high } \\
\text { frequency }\end{array}$ \\
\hline $\begin{array}{l}\text { Litscher. } \\
2006 \text { [12] }\end{array}$ & 9 & $\begin{array}{l}\text { Partially } \\
\text { double } \\
\text { blinded }\end{array}$ & 124 & $\begin{array}{c}\text { GV20, Ex.1, } \\
\text { Ex. 9, He7, Pe6, } \\
\text { CV6, St36, Sp6 }\end{array}$ & None & $\begin{array}{l}\text { Manual and } \\
\text { laser }\end{array}$ & $\begin{array}{l}\text { Spectral } \\
\text { analysis }\end{array}$ & $\begin{array}{c}\text { Significant changes } \\
\text { at sedative points }\end{array}$ \\
\hline $\begin{array}{c}\text { Sakai } \\
2007[15]\end{array}$ & 16 & $\begin{array}{c}\text { Not } \\
\text { blinded }\end{array}$ & 19 & $\begin{array}{c}\text { Ant fibers of } \\
\text { trapezius muscle }\end{array}$ & & & $\begin{array}{l}\text { Spectral analysis } \\
\text { using FFT }\end{array}$ & $\begin{array}{c}\text { Significant } \\
\text { changes }\end{array}$ \\
\hline $\begin{array}{c}\text { Tong, } \\
2008[16]\end{array}$ & 6 & Blinded & 16 & LI4 & Yes & $\begin{array}{l}\text { EA versus } \\
\text { manual }\end{array}$ & $\begin{array}{l}\text { Spectral edge } \\
\text { frequecy } \\
\text { analysis }\end{array}$ & $\begin{array}{c}\text { Significant changes } \\
\text { with manual } \\
\text { acupuncture }\end{array}$ \\
\hline $\begin{array}{l}\text { Streitberger } \\
2008[14]\end{array}$ & 20 & $\begin{array}{l}\text { Single } \\
\text { blinded }\end{array}$ & 124 & LI4 & Yes & Manual & $\begin{array}{l}\text { Spectral } \\
\text { analysis }\end{array}$ & $\begin{array}{l}\text { Significant } \\
\text { changes }\end{array}$ \\
\hline $\begin{array}{c}\text { Chang } \\
2009[10]\end{array}$ & 6 & Blinded & 12 & PC6 & Yes & Manual & $\begin{array}{l}\text { Spectral } \\
\text { analysis }\end{array}$ & $\begin{array}{l}\text { Significant } \\
\text { changes }\end{array}$ \\
\hline
\end{tabular}

EA: Electro-acupuncture; FFT: Fast Fourier transform. 


\section{Contributors}

MTC: Conception and design, acquisition, analysis or interpretation of data, drafting the work, agreement to be accountable for all aspects of the work in ensuring that questions related to the accuracy or integrity of any part of the work are appropriately investigated and resolved.

MKT: Conception and design, acquisition, analysis or interpretation of data, drafting the work, agreement to be accountable for all aspects of the work in ensuring that questions related to the accuracy or integrity of any part of the work are appropriately investigated and resolved.

DY: Conception and design, acquisition, analysis or interpretation of data, drafting the work, agreement to be accountable for all aspects of the work in ensuring that questions related to the accuracy or integrity of any part of the work are appropriately investigated and resolved.

CC: Conception and design, acquisition, analysis or interpretation of data, drafting the work, agreement to be accountable for all aspects of the work in ensuring that questions related to the accuracy or integrity of any part of the work are appropriately investigated and resolved.

\section{Funding}

None.

\section{Competing Interests}

None.

\section{Patient Consent}

Obtained.

\section{Ethical Approval}

The study protocol was approved by the Local Ethics Committee of Baskent University, Faculty of Medicine, Ankara, Turkey.

\section{Editoriall Policy}

"I [Mehmet Tuğrul Cabığlu], the Corresponding Author of this article contained within the original manuscript which includes any diagrams \& photographs and any related or stand alone film submitted (the Contribution”), has the right to grant on behalf of all authors and does grant on behalf of all authors, a licence to the BMJ Publishing Group Ltd and its licensees, to permit this Contribution (if accepted) to be published in any BMJ products and to exploit all subsidiary rights, as set out in our licence set out at:

http://aim.bmj.com/site/about/licence.pdf.

\section{References}

[1] Moffet, H.H. (2006) How Might Acupuncture Work? A Systematic Review of Physiologic Rationales from Clinical Trials. BMC Complementary and Alternative Medicine, 6, 25. http://dx.doi.org/10.1186/1472-6882-6-25

[2] Zhao, Z.Q. (2008) Neural Mechanism Underlying Acupuncture Analgesia. Progress in Neurobiology, 85, 355-375. http://dx.doi.org/10.1016/j.pneurobio.2008.05.004

[3] Napadow, V., Ahn, A., Longhurst, J., et al. (2008) The Status and Future of Acupuncture Mechanism Research. he Journal of Alternative and Complementary Medicined, 14, 861-869. http://dx.doi.org/10.1089/acm.2008.SAR-3

[4] MacPherson, H., Altman, G.D., Hammerschlag, R., et al. (2010) Revised Standards for Reporting Interventions in Clinical Trials of Acupuncture (STRICTA): Extending the CONSORT Statement. PLoS Medicine, 7, e1000261. http://dx.doi.org/10.1371/journal.pmed.1000261

[5] Moffet, H.H. (2008) Traditional Acupuncture Theories Yield Null Outcomes: A Systematic Review of Clinical Trials. Journal of Clinical Epidemiology, 61, 741-747. http://dx.doi.org/10.1016/j.jclinepi.2008.02.013

[6] Hori, E., Takamoto, K., Urakawa, S., et al. (2010) Effects of Acupuncture on the Brain Hemodynamics. Autonomic Neuroscience: Basic and Clinical, 157, 74-80. http://dx.doi.org/10.1016/j.autneu.2010.06.007 
[7] Kagitani, F., Uchida, S. and Hotta, H. (2010) Afferent Nerve Fibers and Acupuncture. Autonomic Neuroscience: Basic and Clinical, 157, 2-8. http://dx.doi.org/10.1016/j.autneu.2010.03.004

[8] Yu, J.S., Zeng, B.Y. and Hsieh, C.L. (2013) Acupuncture Stimulation and Neuroendocrine Regulation. International Review of Neurobiology, 111, 125-40. http://dx.doi.org/10.1016/B978-0-12-411545-3.00006-7

[9] Bai, L.J., Qin, W., Tian, J., et al. (2009) Detection of Dynamic Brain Networks Modulated by Acupuncture Using a Graph Theory Model. Progress in Natural Science, 19, 827-835. http://dx.doi.org/10.1016/j.pnsc.2008.09.009

[10] Chang, S., Chang, Z.G., Li, S.J., et al. (2009) Effects of Acupuncture at Neiguan (PC 6) on Electroencephalogram. Chinese Journal of Physiology, 52, 1-7. http://dx.doi.org/10.4077/CJP.2009.AMG070

[11] Chen, A.C., Liu, F.J., Wang, L. and Arendt-Nielsen, L. (2006) Mode and Site of Acupuncture Modulation in the Human Brain: 3D (124-ch) EEG Power Spectrum Mapping and Source Imaging. Neuroimage, 29, 1080-1091. http://dx.doi.org/10.1016/j.neuroimage.2005.08.066

[12] Litscher, G. (2006) Electroencephalogram—Entropy and Acupuncture. Anesthesia \& Analgesia, 102, 1745-1751. http://dx.doi.org/10.1213/01.ane.0000217188.71710.47

[13] Litscher, G. (2010) Ten Years Evidence-based High-Tech Acupuncture Part 3: A Short Review of Animal Experiments. Evidence-Based Complementary and Alternative Medicine, 7, 151-155. http://dx.doi.org/10.1093/ecam/nen034

[14] Streitberger, K., Steppan, J., Maier, C., et al. (2008) Effects of Verum Acupuncture Compared to Placebo Acupuncture on Quantitative EEG and Heart Rate Variability in Healthy Volunteers. The Journal of Alternative and Complementary Medicine, 14, 505-513. http://dx.doi.org/10.1089/acm.2007.0552

[15] Sakai, S., Hori, E., Umeno, K., et al. (2007) Specific Acupuncture Sensation Correlates with EEGs and Autonomic Changes in Human Subjects. Autonomic Neuroscience: Basic and Clinical, 133, 158-169. http://dx.doi.org/10.1016/j.autneu.2007.01.001

[16] Tong, J.J., Zhu, D.H., Chen, Y.Q., Lv, Y.Y. and Chen, H.D. (2008) Effects of Acupuncture in Pain Relief Based on EEG Research. International Conference on Information Technology and Applications in Biomedicine, Shenzhen, 30-31 May 2008, 578-581. http://dx.doi.org/10.1109/ITAB.2008.4570521

[17] Rosted, P., Griffiths, P.A., Bacon, P. and Gravill, N. (2001) Is There an Effect of Acupuncture on the Resting EEG? Complementary Therapies in Medicine, 9, 77-81. http://dx.doi.org/10.1054/ctim.2001.0435

[18] Xu, M., Tomotake, M., Ikuta, T., Ishimoto, Y. and Okura, M. (1998) The Effects of Qi-Gong and Acupuncture on Human Cerebral Evoked Potentials and Electroencephalogram. The Journal of Medical Investigation, 44, 163-171.

[19] Zhang, G.F., Qu, S.S., Zheng, Y., Chen, J.Q., Deng, G.Z., Yang, C.H. and Huang, Y. (2013) Key Regions of the Cerebral Network Are Altered after Electroacupuncture at the Baihui (GV 20) and Yintang Acupuncture Points in Healthy Volunteers: An Analysis Based on Resting fcMRI. Acupuncture in Medicine, 31, 383-388. http://dx.doi.org/10.1136/acupmed-2012-010301

[20] Chang, S. (2013) The Meridian System and Mechanism of Acupuncture: A Comparative Review. Part 3: Mechanisms of Acupuncture Therapies. Taiwanese Journal of Obstetrics and Gynecology Home, 52, 171-184. http://dx.doi.org/10.1016/j.tjog.2013.04.005

[21] Li, L., Liu, H., Li, Y.-Z., et al. (2008) The Human Brain Response to Acupuncture on Same-Meridian Acupoints: Evidence from an fMRI Study. Journal of Alternative and Complementary Medicine, 14, 673-678. http://dx.doi.org/10.1089/acm.2008.0036

[22] Majumdar, K. (2011) Human Scalp EEG Processing: Various Soft Computing Approaches. Applied Soft Computing, 11, 4433-4437. http://dx.doi.org/10.1016/j.asoc.2011.07.004

[23] Schaul, N. (1998) The Fundamental Neural Mechanisms of Electroencephalography. Electroencephalography and Clinical Neurophysiology, 106, 101-107. http://dx.doi.org/10.1016/S0013-4694(97)00111-9

[24] Zhang, W., Luo, F., Qi, Y., Wang, Y., Chang, J., Woodward, D.J., Chen, A.C. and Han, J. (2003) Modulation of Pain Signal Processing by Electric Acupoint Stimulation: An Electroencephalogram Study. Acta Scientiarum Naturalium Universitatis Pekinensis, 35, 236-240.

[25] Abad-Alegria, F., Pomaròn, C., Aznar, C., Muñoz, C. and Adelantado, S. (2001) Objective Assessment of the Sympatholytic Action of the Nei-Kuan Acupoint. The American Journal of Chinese Medicine, 29, 201-210. http://dx.doi.org/10.1142/S0192415X0100023X 